\title{
Andrea Kloß: Deliberative Offenheit durch Empathie: Eine experimentelle Untersuchung von Unterhaltung im politischen Kontext
}

\author{
Wiesbaden: Springer VS 2020. 166 Seiten. Preis: $€ 49,99$
}

\section{Nicole Podschuweit}

Angenommen: 22. Dezember 2021 / Online publiziert: 24. Januar 2022

(C) Der/die Autor(en) 2022

Mit der Verbreitung des sogenannten Web 2.0 setzte sich alsbald die Erkenntnis durch, dass deliberative Potenziale digitaler Medienplattformen bzw. -formate bei Weitem nicht ausgeschöpft werden. Dies liegt auch daran, dass die Nutzer Diskussionsangebote nicht wahrnehmen oder sich in einer Weise an Diskussionen beteiligen, die einem deliberativen Diskurs abträglich ist. Wie man Diskursteilnehmer (wieder) für Meinungen der Gegenseite öffnen und die Diskussionskultur in unserer Gesellschaft verbessern kann, ist folglich eine dringliche, hoch relevante Frage. Einen Beitrag zu ihrer Beantwortung leistet Andrea Kloß mit diesem Buch, das aus ihrer Dissertation hervorging. Der Ansatz der Autorin ist originell: Ausgangspunkt ist die Idee, dass Unterhaltungsformate die deliberative Offenheit der Rezipienten steigern, indem sie Empathie mit Protagonisten auslösen, die ihr Verhalten gegenüber anderen von einem konfrontativen hin zu einem offenen ändern. Aussichtsreich erscheint dieser Ansatz, weil sich die Botschaft „Sei offen für deine Kontrahenten und ihre Positionen!“ über Unterhaltungsmedien auch politisch weniger Interessierten oder Menschen, die auf gegensätzliche Meinungen mit Skepsis und Ablehnung reagieren, niederschwellig vermitteln lässt.

In der Habermas'schen Tradition geht die Autorin davon aus, dass ein respektvoller Umgang miteinander eine Voraussetzung für problemlösungsorientierte gesellschaftliche Diskurse ist. Ein solcher beinhaltet, dass man sich auch mit Argumenten der Gegenseite auseinandersetzt, statt diese unreflektiert niederzuschmettern. Gemäß der zentralen Annahme der Autorin kann Empathie im Sinne des Mitfühlens mit anderen genau diese Bereitschaft fördern. Die entsprechenden Theoriestränge führt

PD Dr. Nicole Podschuweit ( $₫)$

Institut für Medienforschung, Technische Universität Chemnitz, Straße der

Nationen 12, 09111 Chemnitz, Deutschland

E-Mail: nicole.podschuweit@phil.tu-chemnitz.de

Seminar für Medien- und Kommunikationswissenschaft, Universität Erfurt, Nordhäuser Str. 63, 99089 Erfurt, Deutschland 
die Autorin in ihrer Arbeit stringent zusammen. Insbesondere macht sie plausibel, weshalb unterhaltende Medieninhalte beeinflussen können, wie wir über politische Standpunkte nachdenken, die konträr zu unseren eigenen sind, und wie wir mit Menschen umgehen, die diese Standpunkte vertreten. Einen fruchtbaren Ansatzpunkt liefern Befunde, nach denen ein eudaimonisches Unterhaltungserleben eine Erweiterung der eigenen (politischen) Perspektive bewirken kann. Ein derart tiefgehendes emotionales Involvement kann z. B. durch „Transformationsgeschichten“ ausgelöst werden. So können Protagonisten, die sich Mitgliedern von Fremdgruppen gegenüber zunächst ablehnend zeigen, sich dann aber zu offenen Interaktionspartnern wandeln, als Vorbilder für die Rezipienten fungieren. Obgleich die theoretischen Ausführungen insgesamt überzeugen, werden zwei Fragen nicht hinlänglich beantwortet: Erstens, inwieweit können wir dem Ideal eines deliberativen Austauschs realiter gerecht werden? Zweitens, weshalb wird der interpersonale Austausch, der nach Darstellung der Autorin essentiell für die Überprüfung ihrer zentralen Annahme ist, nur am Rande behandelt?

Die relevante Zielgröße des theoretischen Modells der Autorin ist deliberative Offenheit. Sie gilt als Voraussetzung für Deliberation als komplexem Konstrukt, das in vier Komponenten aufgespalten wird: 1) allgemeine Offenheit sowie 2) Offenheit gegenüber verschiedenen Standpunkten zu einem spezifischen kontroversen Thema, 3) dazu vertretenen Argumenten beider Seiten sowie 4) der Beteiligung an einer Diskussion mit Menschen, die einen anderen Standpunkt dazu vertreten als man selbst. Gemäß dem Modell fördern Transformationsgeschichten deliberative Offenheit direkt und indirekt, indem sie das Empathieempfinden der Rezipienten für ,gegensätzliche“ Charaktere steigern. Denkbar ist aber auch, dass Transformationsgeschichten Reaktanz hervorrufen und deliberative Offenheit hemmen. Das Modell der Autorin ist in sich stimmig, scheint jedoch vorrangig mit Blick auf die Operationalisierung und statistische Überprüfung der wenigen daraus abgeleiteten Hypothesen entwickelt worden zu sein. Die umfassenden theoretischen Vorüberlegungen spiegeln sich darin nur begrenzt wider. Ausgehend davon wäre das stark reduzierte Modell eher als Element eines umfassenderen Modells zu denken, das sowohl das interpersonale Kommunikationsverhalten als Manifestation deliberativer Offenheit beinhaltet, als auch weitere relevante Erklärgrößen (etwa allgemeines Kommunikationsverhalten der Rezipienten, Themen-Involvement, individuelle bzw. (Mehrheits-)Meinung ihrer sozialen Bezugsgruppen zum jeweiligen Thema).

Ihre Hypothesen testet die Autorin durch experimentell angelegte Online-Befragungen zweier über das SoSci-Panel rekrutierter Stichproben. Als Stimulus dienen in Spielfilmen (Dallas Buyers Club bzw. Men of Honor) erzählte Transformationsgeschichten, die sich nach ihrem Modell auf die Aufgeschlossenheit der Rezipienten gegenüber Sichtweisen zu damit in Verbindung stehenden, kontroverse Themen (Diversität bzw. Zuwanderung) auswirken sollten. (In der Kontrollbedingung erlebten die Befragten den Protagonisten jeweils nur konfrontativ und nicht versöhnlich.) Ihr Filmmaterial hat die Autorin umsichtig ausgewählt, aufbereitet und durch eine qualitative Vorstudie auf seine Eignung überprüft. Die Operationalisierung der nach dem Modell relevanten Größen ist ebenso plausibel wie die Auswahl der Analysestrategie. Nicht ganz nachvollziehbar ist, weshalb zwei Stichproben - eine höher und eine niedriger gebildete - erforderlich waren bzw. weshalb eine Stichprobe, 
in der unterschiedliche Bildungsschichten repräsentiert sind, nicht ausreichend war. Mit Blick auf die Auswahlgesamtheit stellt sich außerdem die Frage, inwieweit Personen, die sich deliberativen Diskursen verweigern bzw. diese sabotieren, im SoSciPanel repräsentiert sind.

Im Ergebnis legen die beiden Umfrageexperimente nahe, dass Transformationsgeschichten die deliberative Offenheit der Rezipienten gegenüber „,gegensätzlichen“ Charakteren direkt (höher gebildete Stichprobe) oder vermittelt über Empathie (beide Stichproben) erhöhen können. Reaktanz lösten die Transformationsgeschichten bei den Befragten nicht aus, was umso mehr dafür spricht, dass sie sich als wirksames Mittel zur Förderung deliberativer Offenheit erweisen können. Dass sich dies in einer erhöhten Bereitschaft zur Teilnahme an Diskussionen mit Anhängern der gegnerischen Seite niederschlägt, kann auf Basis der Studie von Andrea Kloß nicht als gesichert gelten. Unabhängig davon geben die Befunde in der Gesamtschau Anlass zum Optimismus, dass Unterhaltungsformate erwünschte Nebenwirkungen auf das gesellschaftliche Miteinander und unsere Diskussionskultur haben können. Als anschlussfähig für weitere Forschung erweist sich die Arbeit von Andrea Kloß auch, weil die Überprüfung von Einflüssen relevanter Erklärgrößen und insbesondere von Effekten unterhaltender Medieninhalte wie Transformationsgeschichten auf das anschließende Diskussionsverhalten noch aussteht. In diesem Zusammenhang - hierauf verweist die Autorin selbst in der Ergebnisdiskussion - stellt sich die Frage nach der Nachhaltigkeit derartiger Effekte bzw. ihrem Transfer auf Diskussionsthemen, denen Rezipienten in ihrem Alltag begegnen. Darüber hinaus ist zu überprüfen, wie resistent solche Effekte gegenüber sozialen Einflüssen sind. So dürften sich in unserem sozialen Umfeld nicht minder relevante „Vorbilder“ finden, an denen wir unser (interpersonales) Kommunikationsverhalten orientieren. Zu guter Letzt gilt es mit Blick auf den praktischen Nutzwert zu ergründen, ob bzw. wie man Unterhaltungsinhalte gezielt einsetzen kann, um die Empathie und die deliberative Offenheit jener Menschen zu erhöhen, die in diesen Bereichen Defizite haben.

Funding Open Access funding enabled and organized by Projekt DEAL.

Open Access Dieser Artikel wird unter der Creative Commons Namensnennung 4.0 International Lizenz veröffentlicht, welche die Nutzung, Vervielfältigung, Bearbeitung, Verbreitung und Wiedergabe in jeglichem Medium und Format erlaubt, sofern Sie den/die ursprünglichen Autor(en) und die Quelle ordnungsgemäß nennen, einen Link zur Creative Commons Lizenz beifügen und angeben, ob Änderungen vorgenommen wurden.

Die in diesem Artikel enthaltenen Bilder und sonstiges Drittmaterial unterliegen ebenfalls der genannten Creative Commons Lizenz, sofern sich aus der Abbildungslegende nichts anderes ergibt. Sofern das betreffende Material nicht unter der genannten Creative Commons Lizenz steht und die betreffende Handlung nicht nach gesetzlichen Vorschriften erlaubt ist, ist für die oben aufgeführten Weiterverwendungen des Materials die Einwilligung des jeweiligen Rechteinhabers einzuholen.

Weitere Details zur Lizenz entnehmen Sie bitte der Lizenzinformation auf http://creativecommons.org/ licenses/by/4.0/deed.de.

PD Dr. Nicole Podschuweit vertritt aktuell eine Professur für Kommunikations- und Medienwissenschaft an der Technischen Universität Chemnitz. An der Universität Erfurt leitet sie das dortige Teilprojekt im BMBF-Verbundprojekt „Journalist*innen und ihr Publikum im digitalen Zeitalter“. 